\title{
Poorly Understood Pelvic Venous Disorders Require a Multidisciplinary Approach
}

\section{Unzureichend erforschte Erkrankungen der Beckenvenen erfordern einen fachübergreifenden Ansatz}

Author

Suat Doganci

Affiliation

Health Sciences University, Gulhane School of Medicine, Department of Cardiovascular Surgery Ankara/Turkey

Key words

pelvic venous disease, pelvic congestion, chronic pelvic pain

Schlüsselwörter

Beckenvenenerkrankung, Beckenstauung, chronische

Beckenschmerzen

published online 05.07.2021

Bibliography

Phlebologie 2021; 50: 279-282

DOI 10.1055/a-1521-5995

ISSN 0939-978X

(c) 2021. Thieme. All rights reserved.

Georg Thieme Verlag KG, Rüdigerstraße 14,

70469 Stuttgart, Germany

Correspondence

Prof. Suat Doganci, MD

Gulhane Egitim ve Arastirma Hastanesi

Kalp Damar Cerrahisi, AD 06018 Etlik/Ankara, Turkey

suat_doganci@yahoo.com

\section{ZUSAMMENFASSUNG}

Beckenvenenerkrankungen umfassen alle chronischen Krankheitsbilder der Beckenvenen, die durch venöse Hypertonie und retrograden Fluss in den Beckenvenen verursacht werden. Sie sind eine häufig ungeklärte und oft unterdiagnostizierte Ursache für chronische Beckenschmerzen bei fortpflanzungsfähigen Frauen. Beckenvenenerkrankungen sind Teil eines fachübergreifenden Problems, das ganzheitlich betrachtet werden sollte. Die neu eingeführte SVP-Klassifikation kann dabei helfen, Patienten korrekt einzuordnen und eine einheitliche Nomenklatur zu verwenden. Endovaskuläre Behandlungsmethoden sind äußerst wirksam und liefern gute Langzeitergebnisse. Patientinnen mit unklaren gynäkologischen Untersuchungsbefunden sollten zur Abklärung von Beckenvenenerkrankungen an einen Spezialisten überwiesen werden.

\section{ABSTRACT}

Pelvic venous diseases encompasses all chronic pathologies of pelvic veins caused by venous hypertension and retrograde flow in pelvic veins. It is commonly unexplained and often underdiagnosed cause of chronic pelvic pain in women of productive age. Pelvic venous pathologies are a part of multidisciplinary problem that should be considered in a holistic approach. Newly offered SVP classification may help correctly classifying patients and using the same nomenclature. Endovascular treatment options are highly effective with good long-term results. Patients with inconclusive gynecologists examinations should be referred to a specialist for the investigation of pelvic venous disorders.

\section{Definition and epidemiology}

The term of pelvic venous diseases (PeVD) encompasses all chronic pathologies of pelvic veins caused by venous retrograde flow in pelvic veins and hypertension. PeVD is a cause of unexplained and often underdiagnosed chronic pelvic pain in women of productive age. Chronic pelvic pain is non-cyclical pain in lower abdomen and pelvis, that lasts longer than six months. In VEIN-TERM Transatlantic Interdisciplinary Consensus Document, venous disease originated chronic pelvic pain is defined as "pelvic pain caused by ovarian and/or pelvic vein reflux and/or obstruction, perineal heaviness, frequent urination, and chronic symptoms that may include postintercourse pain and be associated with vulvar, perineal, and/or lower extremity varicose veins" [1].

According to a study, among 100 vascular specialists $9 \%$ responded that they did not consider pelvic vein reflux as a pathological entity and $11 \%$ stated that they never investigate or treat it [2]. Although it is estimated that chronic pelvic pain accounts for $40 \%$ of gynecological consultations, it is predicted that half of these patients remain untreated for years without an accurate diagnosis [3]. It was also found that $10 \%$ of healthy women had ovarian vein reflux and $60 \%$ of them had PeVD [4]. True preva- 
lence of PeVD is still uncertain; however, studies have shown that $30 \%$ of patients with chronic pelvic pain have PeVD as sole cause of their pain and an additional $15 \%$ have PeVD along with another pelvic pathology [5].

\section{Etiology and pathophysiology}

The exact etiology of PeVD is unknown. Multiple factors such as valvular incompetence, venous obstruction and hormones may play an important role in congestion or slow drainage of uteroovarian and salpingo-ovarian veins. The release of pain-triggering mediators due to dilatation of veins, caused by venous stasis, is a possible cause of pain in PeVD [6]. On the other hand, hormonal changes (high levels of estrogen and progesterone) during pregnancy cause vasodilation of ovarian veins, resulting in a $60 \%$ increase in capacity of pelvic venous system. Reduction of symptoms after menopause indicates effect of hormones on pelvic congestion syndrome (PCS) [7].

Two anatomical signs can define PeVD: ovarian vein reflux and pelvic varicose veins. Term of primary pelvic insufficiency includes congenital or acquired ovarian vein reflux from non-obstructive causes. In an autopsy series, congenital absence of ovarian vein valves on the right and left sides have been reported in $6 \%$ and $13 \%-15 \%$ of the patients, respectively. Valvular incompetence was also observed among 35-46\% of right ovarian veins and $41-43 \%$ of left ovarian veins [8]. Secondary pelvic insufficiency includes pathologies involving collateral veins formed by ovarian and pelvic venous insufficiency such as Nutcracker syndrome (left renal vein compression by superior mesenteric artery), MayThurner syndrome (left iliac vein compression by right common iliac artery) or post-thrombotic occlusion of iliac vein. Segmental overload in veins can also cause venous obstruction. This overload may be due to left renal vein thrombosis (with Renal Cell Carcinoma), intravascular tumor thrombosis in inferior vena cava (IVC), cirrhosis, congenital vascular malformations and retro aortic left renal vein [9].

\section{Diagnosis}

A comprehensive background information and physical examination should be performed for evaluation of chronic pelvic pain. Postural changes, non-cyclic pelvic pain exacerbated by walking or/and sexual intercourse, and menstruation is main clinical symptoms of PeVD. There are four major clinical manifestations [1]:

I. Prolonged (3-6 months) non-cyclical chronic pelvic pain with dyspareunia, dysmenorrhea and dysuria.

II. Presence of varicose veins in perineal, vulvar and gluteal regions. III. Renal symptoms accompanied by flank pain hematuria and proteinuria.

IV. Leg pain presenting with swelling and varicose veins.

In a study conducted by Mahmoud et al. [9] it was reported that the most common finding was dysmenorrhea (86\%). Other common symptoms are lower extremity varicose veins (58.7\%), vulvar varicosities (45.9\%) and dyspareunia (40.8\%). In another study, in accordance with results of pelvic venography, clinical signs with the highest sensitivity for diagnosis of PCS are vulvar varicose veins $(100 \%)$, sensitivity of the ovarian region upon palpation (87\%), dysmenorrhea (84\%) and postcoital pain (79\%) [10]. It should be kept in mind that if patient also has a history of deep vein thrombosis in lower extremity, symptoms of PeVD may occur because of post-thrombotic syndrome caused by iliac vein obstruction [11].

Imaging methods are important in patients with clinically suspected PeVD. Non-invasive imaging methods such as ultrasonography (USG), magnetic resonance imaging (MRI) and computed tomography (CT) are recommended as first line of radiological examination. These imaging methods are also used to diagnose additional pelvic pathologies associated with PeVD. Use of intravascular USG in diagnosis of PeVD is very rare. It is mostly applied to diagnose compressive syndromes. However, venography remains as gold standard method in diagnosis [12].

Trans-abdominal USG continues to be first-line imaging method for evaluating pelvic pain. When applied by experienced specialists, it can consistently visualize ovarian vascular structures, vein diameters and possible reflux, considering all potential pathologies that may contribute to PeVD. Trans-abdominal USG can avoid other expensive and time-consuming imaging modalities that might require ionizing radiation or intravenous contrast. Major limitations of the examination method include images being hindered by intestinal gas, inferior image quality in obese patients and demand of an experienced specialist. It has been emphasized that sensitivity and specificity of trans-abdominal USG in evaluating ovarian vein insufficiency is respectively $100 \%$ and $75 \%$ [13].

Trans-vaginal USG provides superior visualization of pelvic venous plexus and is not affected by the patient's position or intestinal gas. The technique also facilitates good visualization of distal ovarian veins and internal iliac veins. Both physiological venous reflux in pelvis, anal canal, vagina, vulva and pathological venous reflux causing varicose veins can be detected. Therefore, it has been suggested that trans-vaginal USG should be a gold standard method in patient follow-up, especially after pelvic vein embolization [13]. Disadvantage of this diagnostic method is that it doesn't provide information about common iliac vein and proximal segment of ovarian vein. If any compression syndromes, such as Nutcracker syndrome and May-Thurner syndrome, is suspected, further imaging may be required using a trans-abdominal Doppler USG or another specific method. Consent of the patient is also one of the most important limitations. In initial trans-vaginal USG examination for diagnosing the etiology of pelvic pain in women who are not pregnant, following diagnostic criteria should be used for PCS [14].

1. Tortuous parametrial/adnexal pelvic veins larger than $4 \mathrm{~mm}$ in diameter

2. Tortuous and dilated arcuate veins in myometrium and involvement of a connection with adnexal varicose veins

3. Decrease in velocity of blood flow $(>3 \mathrm{~cm} / \mathrm{s}$ ) or reflux in left ovarian vein with Valsalva maneuver

4. Polycystic ovary sign not associated with amenorrhea or hirsutism (These findings are present in $50 \%$ of patients)

While dilatation over $8 \mathrm{~mm}$ in diameter of pelvic veins is associated with pathological reflux and symptoms, 4-8 mm diameter 
has been linked to asymptomatic reflux. In a study, it was shown that diameter of left ovarian vein in patients with PeVD was significantly larger in symptomatic individuals when compared to healthy individuals [14]. However, Dos Santos et al. [15] found no difference in diameter between competent and refluxing ovarian veins (7.2 mm (3-13); $8.5 \mathrm{~mm}(4-13), p=0.2)$. Therefore, it has been claimed that considering the diameter as a sign of ovarian venous reflux would not be an appropriate approach. In recently published SVP (Symptom-Varices-Pathophysiology) classification, it has been stated that incidence of venous disease increases with increase in diameter of vein, but the classification remained cautious about presenting a clarification regarding this situation [11].

Lower extremity venous doppler USG is an essential part of imaging protocol for evaluation of PCS, especially in presence of atypical varicose veins. Atypical varicose veins on back and lateral sides of thigh in patients without reflux at saphenofemoral junction are highly pathognomonic for pelvic venous insufficiency [13].

Computed tomography and MRI allow detailed anatomical examination of pelvic vascular structures and surrounding tissues, helping to analyze extra-luminal venous compressions more comprehensively [16]. Among cross-sectional imaging methods, CT has less diagnostic importance than others. CT cannot be used as a routine diagnostic method like USG, and it does not provide sufficient information about hemodynamic changes in pelvic veins [7]. Venous reflux can be difficult to detect by $C T$, if no additional attention is paid to bolus injection timing. CT helps differential diagnosis in pelvic pathologies. MRI, with phase contrast technique, can optimize evaluation of reflux. MRI provides effective identification of pelvic varicose veins without exposure to radiation, with a specificity of up to $67-75 \%$ and a sensitivity of up to $100 \%$ compared to venography. CT and MRI are superior to USG in evaluation of tortuous, enlarged pelvic and ovarian varicose veins [3]. However, diagnostic quality may be decreased because of insufficient filling of pelvic varices, since MRI is performed while patient is in supine position. Moreover, MRI is not used in followup due to metallic coil artifacts after embolization [16].

Retrograde selective venography of ovarian and internal iliac veins for diagnosis of pelvic venous pathologies continues to remain gold standard imaging method. Venography is performed for confirmation of diagnosis, evaluation of venous anatomy and collateral venous circulation, and decision of embolization [5].

Due to Trendelenburg position, $\mathrm{CO}_{2}$ insufflation of peritoneal cavity, adhesions, application in supine position and some other disadvantages, diagnostic laparoscopy has a sensitivity around $40 \%$. It also gives negative results in $80-90 \%$ of PeVD patients [4].

\section{Classification of PeVD (SVP Classification)}

Use of previously nomenclature for pelvic venous disorders fails to describe complex and interrelated pelvic venous circulation, further causing misdiagnosis and poor treatment outcomes. Classifications for PeVD have been suggested in literature. Definition of homogenous patient population, development of equipment to investigate treatment and planning of scientific clinical trials are required in order to optimize clinical care of patients with PeVD. Therefore, recently published SVP classification was designed as an initial point to clarify the classification of PeVD. The SVP classification suggested for PeVD consists of three parts: symptoms (S), varices (V) and pathophysiology (P). Pathophysiology $(P)$ domain encompasses pelvic and abdominal vein anatomy $(A)$, symptomrelated hemodynamic abnormalities $(\mathrm{H})$ and underlying etiology (E). According to this classification, the pelvic disease score of an individual is shown as $\mathrm{SVP}_{\mathrm{A}, \mathrm{H}, \mathrm{E}}[11]$.

In pelvic venous classification, patient's clinical symptoms (" $S$ ") domain is determined by subscripts ranging from 0 to 3 . This section is arranged according to anatomical regions descending from renal veins to lower extremities. Venous system of pelvis can be thought as comprising three reservoirs in which varicose veins can develop: (i) renal hilum, (ii) venous plexuses of the pelvis, and (iii) extra-pelvic vessels of pelvic origin. Lower extremity vessels contain a fourth reservoir that has a relation with extra-pelvic varicose veins of pelvic origin. However, lower extremity reservoir is best described by CEAP classification (Clinical Etiological Anatomical Pathophysiological Classification) and is not included in SVP classification. Pathophysiology domain $(P)$ is a combination of anatomical $(A)$, hemodynamic $(H)$, and etiological $(E)$ subfields. Anatomical parts of abdomen and pelvis are indicated with anatomical abbreviations. Underlying hemodynamic $(\mathrm{H})$ irregularities reflux $(R)$, obstruction $(O)$ or both $(R, O)$ are shown. The etiology of pelvic venous pathology $\left(P_{E}\right)$ is defined as thrombotic $(T)$, nonthrombotic (NT), or congenital (C). All three components are indicated by a subscript in " $P$ " category $\left(\mathrm{P}_{\mathrm{E}, \mathrm{T}, \mathrm{NT}}\right)[11]$.

\section{Treatment}

\section{Medical Treatment}

Medical treatment for PCS syndrome includes physiotherapy, analgesics, non-steroidal anti-inflammatory drugs (NSAIDs), dihydroergotamine, progestins (contraceptives, hormone replacement therapy, danazol), gonadotropin-releasing hormone $(\mathrm{GnRH})$ agonists, and venoactive drugs. Since pathophysiology of PCS is believed to occur because of both hormonal and mechanical reasons, induction of a hypoestrogenic state through hormonal suppression of ovary and/or vasoconstriction is primary goal of medical therapy. However, medical therapy has not been shown to be effective in long-term treatment. Studies have shown that NSAIDs, medroxyprogesterone acetate, GnRH agonists, and etonogestrel implants provide only temporary pain relief to PeVD patients. Intravenous dihydroergotamine is thought to reduce pain by creating vasoconstriction in uterine and parametrium veins but is not used in routine treatment due to its short-acting nature and adverse effects. In many studies, micronized purified flavonoid fraction has been shown to provide significant pain reduction and improvement in venous outflow within 2-4 weeks of treatment initiation. Studies related to the medical therapy of PeVD do not provide sufficient evidence for treatment efficacy because randomized controlled studies conducted by using unverified criteria on small groups of patients [17]. In addition, medical therapy has not been shown to be effective in long-term treatment. 


\section{Endovascular Treatment}

Since 1993, when Edwards et al.[18] described first bilateral ovarian vein embolization for treatment of PeVD, this method has become mainstay for treating PeVD caused by gonadal and pelvic vein insufficiency. Although transfemoral or trans-jugular approach is frequently used to provide ovarian and internal iliac vein access, basilic and cephalic veins can also be used for vascular access. Various materials can be used for the embolization, including sclerosing foam, adhesives, amplatzer vascular plugs, and coils.

Transcatheter embolization is performed after gonadal venous insufficiency is confirmed by venography. Complete embolization of all collateral vessels associated with lateral branches of ovarian veins and opposite side of pelvis is required for clinical success. Embolization should be applied up to a distance of $3 \mathrm{~cm}$ from junction of renal vein on left side and to IVC with the lateral branches of the ovarian veins on right. In order to increase treatment success, $3 \%$ sodium tetradecyl sulfate or foam sclerosants such as $5 \%$ sodium morrhuate are used, besides coil embolization [19].

Vascular access and embolization should be done carefully to prevent serious complications. Patients might present complications related to coil embolization such as vein perforation ( $1 \%)$, transient pain in those who received additional sclerotherapy (8-100\%), transient fever (12\%), superficial thrombophlebitis at intervention site $(9 \%)$, and coil migration ( $<2 \%)$. A study reported that post-thromboembolic syndrome with pelvic pain, tenderness along the vein in which embolization was applied, and fever reaching $37.5^{\circ} \mathrm{C}-37.8^{\circ} \mathrm{C}$ was seen in $20 \%$ of the patients who underwent embolization with nickel-chromium coils [19].

Many studies have investigated effectiveness and safety of coil embolization treatment, and it has been reported that the average success rate is around 75\%.[19] Even though studies have reported high success rates following endovascular embolization, evidence obtained in these studies have poor quality because of patient population, inclusion criteria, variety of procedures performed and lack of standardization in diagnostic methods used to evaluate the results. In a study evaluating vascular plugs, despite the advantage of less exposure to radiation, no significant difference was found in clinical success and improvement in 1-year follow-up results. In patients who underwent embolization with ethylene vinyl alcohol copolymer material, a success rate of $76.4 \%$ was observed and no complications were found [20].

It is known that venous occlusive diseases such as Nutcracker syndrome and May-Turner syndrome cause PCS.It has been observed that symptoms of pelvic venous insufficiency improved after stenting of occluded vein. However, randomized controlled studies evaluating the efficacy of stenting are needed [11].

\section{Conflict of Interest}

The authors declare that they have no conflict of interest.

\section{References}

[1] Eklof B, Perrin M, Delis KT et al. American Venous Forum; European Venous Forum; International Union of Phlebology; American College of Phlebology; International Union of Angiology. Updated terminology of chronic venous disorders: the VEIN-TERM transatlantic interdisciplinary consensus document. J Vasc Surg 2009; 49: 498-501

[2] Campbell B, Goodyear S, Franklin I et al. Investigation and treatment of pelvic vein reflux associated with varicose veins: Current views and practice of 100 UK vascular specialists. Phlebology 2020; 35: 56-61

[3] Borghi C, Dell'Atti L. Pelvic congestion syndrome: the current state of the literature. Arch Gynecol Obstet 2016; 293: 291-301

[4] Belenky A, Bartal G, Atar E et al. Ovarian varices in healthy female kidney donors: incidence, morbidity, and clinical outcome. Am J Roentgenol 2002; 179: 625-627

[5] Lazarashvili Z, Antignani PL, Monedero JL. Pelvic congestion syndrome: prevalence and quality of life. Phlebolymphology 2016; 23: 123-126

[6] Soysal ME, Soysal S, Vicdan K et al. A randomized controlled trial of goserelin and medroxyprogesterone acetate in the treatment of pelvic congestion. Hum Reprod 2001; 16: 931-939

[7] Antignani PL, Lazarashvili Z, Monedero JL et al. Diagnosis and treatment of pelvic congestion syndrome: UIP consensus document. Int Angiol 2019; 38: 265-283

[8] Winer AG, Chakiryan NH, Mooney RP et al. Secondary pelvic congestion syndrome: description and radiographic diagnosis. Can J Urol 2014; 21 : 7365-7368

[9] Mahmoud O, Vikatmaa P, Aho P et al. Efficacy of endovascular treatment for pelvic congestion syndrome. J Vasc Surg Venous Lymphat Disord 2016; 4: 355-370

[10] Herrera-Betancourt AL, Villegas-Echeverri JD, López-Jaramillo JD et al. Sensitivity and specificity of clinical findings for the diagnosis of pelvic congestion syndrome in women with chronic pelvic pain. Phlebology 2018; 33: 303-308

[11] Meissner MH, Khilnani NM, Labropoulos $\mathrm{N}$ et al. The symptoms- varicespathophysiology (SVP) classification of pelvic venous disorders a report of the american vein \& lymphatic society international working group on pelvic venous disorders. J Vasc Surg Venous Lymphat Disord 2021: S2213 333X(21)00071-8

[12] Ganeshan A, Upponi S, Hon LQ et al. Chronic pelvic pain due to pelvic congestion syndrome: the role of diagnostic and interventional radiology. Cardiovasc Intervent Radiol 2007; 30: 1105-1111

[13] Park SJ, Lim JW, Ko YT et al. Diagnosis of pelvic congestion syndrome using transabdominal and transvaginal sonography. Am J Roentgenol 2004; 182: 683-688

[14] Whiteley MS, Dos Santos SJ, Harrison CC et al. Transvaginal duplex ultrasonography appears to be the gold standard investigation for the haemodynamic evaluation of pelvic venous reflux in the ovarian and internal iliac veins in women. Phlebology 2015; 30: 706

[15] Dos Santos SJ, Holdstock JM, Harrison CC et al. Ovarian vein diameter cannot be used as an indicator of ovarian venous reflux. Eur J Vasc Endovasc Surg 2015; 49: 90-94

[16] Arnoldussen CW, de Wolf MA, Wittens $\mathrm{CH}$. Diagnostic imaging of pelvic congestive syndrome. Phlebology 2015; 30 (Suppl. 1): 67-72

[17] Brown CL, Rizer M, Alexander R et al. Pelvic Congestion Syndrome: Systematic Review of Treatment Success. Semin Intervent Radiol 2018; 35: $35-40$

[18] Edwards RD, Robertson IR, MacLean AB et al. Case report: pelvic pain syndrome-successful treatment of a case by ovarian vein embolization. Clin Radiol 1993; 47: 429-431

[19] Whiteley MS, Lewis-Shiell C, Bishop SI et al. Pelvic vein embolisation of gonadal and internal iliac veins can be performed safely and with good technical results in an ambulatory vein clinic, under local anaesthetic alone - Results from two years' experience. Phlebology 2018; 33: 575-579

[20] Marcelin C, Izaaryene J, Castelli M et al. Embolization of ovarian vein for pelvic congestion syndrome with ethylene vinyl alcohol copolymer (Onyx). Diagn Interv Imaging 2017; 98: 843-848 